\title{
Terapia comportamental associada a neuromodulação no tratamento da bexiga e intestino em indivíduos com Parkinson: um estudo piloto
}

\author{
Behavioral therapy associated with neuromodulation \\ in the treatment of bladder and bowel in individuals \\ with Parkinson's: a pilot study
}

\author{
Maria Aneilma Ribeiro Azevedo ${ }^{1}$ (1) \\ Lilian Lira Lisboa ${ }^{2}$ (1) \\ Cléa Emanuela Barreto de Medeiros $^{3}$ (1) \\ Valéria Azevedo de Almeida 4 (1) \\ Rafael Pauletti Gonçalves ${ }^{5}$ (b)
}

\begin{abstract}
${ }^{1}$ Autora para correspondência. Instituto de Ensino e Pesquisa Alberto Santos Dumont (Macaíba). Rio Grande do Norte, Brasil. aneilmaa@hotmail.com ${ }^{2}$ Universidade Federal do Rio Grande do Norte (Natal), Instituto de Ensino e Pesquisa Alberto Santos Dumont (Macaíba). Rio Grande do Norte, Brasil. lilian.lisboa@isd.org.br

3-5Instituto de Ensino e Pesquisa Alberto Santos Dumont (Macaíba). Rio Grande do Norte, Brasil. clea@edu.isd.org.br, valeria@edu.isd.org.br, rafael.goncalves@isd.org.br
\end{abstract}

RESUMO | OBJETIVO: O objetivo deste estudo foi avaliar os efeitos da terapia comportamental associada à neuromodulação na bexiga e intestino neurogênicos na Doença de Parkinson. MÉTODOS: Trata-se de um estudo piloto. Os participantes, após assinarem o termo de consentimento, foram submetidos à avaliação das funções eliminatórias, qualidade de vida e estágio da doença por meio de instrumentos específicos. Os sujeitos realizaram dezesseis sessões de neuromodulação parassacral e acompanhamento da terapêutica comportamental. RESULTADOS: Na reavaliação apresentaram melhoras dos sintomas urinários, evacuatórios e dos domínios de qualidade de vida. Foi apresentado também aumento da capacidade vesical que embora não significativos já expressam melhora do quadro clínico expresso pelo paciente. Também observou-se melhora para frequência evacuatória, consistência das fezes e eliminação do esforço para evacuar. CONCLUSÃo: Sugere-se que a neuromodulação acrescida da terapêutica comportamental é uma técnica de baixo custo e com resultados significativos para os sintomas urinários e melhora dos valores de atividade de vida diária, bem-estar emocional, estigma, suporte social, cognição, comunicação e desconforto corporal medidos para qualidade de vida.

DESCRITORES: Doença de Parkinson. Estimulação Elétrica Nervosa Transcutânea. Incontinência urinária. Constipação.

\begin{abstract}
AIMS: The study aims to evaluate the effects of behavioral therapy associated with neuromodulation at neurogenic bladder and bowel on Parkinson's disease. METHODS: It is a pilot study. After signing consent forms, the participants had an evaluation of elimination functions, quality of life, and Parkinson's staging through specific instruments. Subjects had sixteen sessions of parasacral neuromodulation and cognitive behavioral therapy attendance. RESULTS: Revaluation showed improvement of urinary and evacuatory symptoms and quality of life domain. There was also an increase in bladder capacity which, although not significant, expresses improvement in the clinical condition expressed by the patient. Improvements at evacuatory frequency, feces consistency, and evacuation strain were also found. CONCLUSION: The study results suggest that neuromodulation associated with behavioral therapy is a low-cost procedure that shows significant results to urinary symptoms and improvements to the quality of life measurements to domains of activities of daily living, emotional well-being, stigma, social support, cognition, communication, and bodily discomfort.
\end{abstract}

KEYWORDS: Parkinson Disease. Transcutaneous Electric Nerve Stimulation. Urinary Incontinence. Constipation. 


\section{Introdução}

A doença de Parkinson (DP) é considerada um distúrbio de natureza neurodegenerativa, progressiva e irreversível, caracterizada pela perda de neurônios dopaminérgicos nos gânglios da base ${ }^{1}$. Os indivíduos com DP podem apresentar uma série de sinais e sintomas motores, como: tremor de repouso, rigidez muscular e bradicinesia, os quais formam a tríade motora clássica². Entretanto, o curso clínico da doença não é restrito apenas aos sintomas motores, incluise também distúrbios do sono, neuropsiquiátricos, autonômicos (urogenital, gastrointestinal, cardiovascular e respiratório), sensoriais e dor ${ }^{3}$. Estes sintomas não motores têm sido apontados como anteriores às disfunções motoras, representando uma fase préclínica que iniciam aproximadamente 20 anos antes que o diagnóstico de DP seja estabelecido, e tendem a piorar com a progressão da doença $a^{4}$.

Apesar do seu significado clínico, esses sintomas são frequentemente negligenciados ou confundidos com sintomas natos do envelhecimento 5 , a exemplo, os sintomas do trato urinário inferior (STUI) e da constipação intestinal $(\mathrm{Cl})$. Geralmente, em pacientes com DP, as queixas urinárias primárias incluem urgência, aumento da frequência, incontinência urinária (IU) e noctúria. Esses sintomas de armazenamento urinário são queixas prevalentes de 57-83\% dos pacientes com DP $\underline{6}$.

A International Continence Society (ICS) define como bexiga hiperativa um conjunto desses sintomas de armazenamento, e quando há uma condição neurológica relevante, a condição é chamada de bexiga neurogênica e é investigada por observação urodinâmica. 6 Os estudos urodinâmicos demonstram capacidade reduzida da bexiga e hiperatividade do detrusor (HD) em $43-93 \%$ dos pacientes com DP ${ }^{7}$. No que diz respeito às queixas intestinais, a constipação apresenta-se como sintoma gastrointestinal mais comum na DP, com uma prevalência estimada em aproximadamente $50 \%$. A constipação é um sintoma subjetivamente percebido, incluindo manifestações como fezes duras, movimentos intestinais reduzidos, inchaço, dor abdominal e esforço durante a defecação ${ }^{8}$.
Embora seja alta a prevalência dos STUI e $\mathrm{Cl}$ e seus impactos na qualidade de vida, as opções de tratamento são atualmente limitadas e, muitas vezes, são pouco toleradas ou ineficazes na DP. Entretanto, intervenções no estilo de vida e modificações comportamentais (investigação dos hábitos de vida do paciente, orientação quanto ao posicionamento ao evacuar, técnicas de respiração, ingestão hídrica adequada, massagem terapêutica abdominal e prática de exercícios físicos) podem atenuar os sintomas, como também neuromodulação (NMS) ${ }^{9}$.

Teorias sugerem que a NMS bloqueia estímulos que causam a hiperatividade do detrusor originados no assoalho pélvico e na bexiga e, desse modo, restaura o equilíbrio normal da micção. Blok et al. (apud Sacomani e Goldman) mostraram que a NMS altera a resposta cortical e atua na modulação da atividade cerebral de áreas relacionadas ao controle sensitivomotor envolvidas no início da micção e sensação de enchimento vesical ${ }^{10}$.

É importante estudar o efeito da NMS associada a terapêutica comportamental pelo impacto significativo na qualidade de vida que a BN e IN causam, podendo levar a limitação de atividades, bem como vergonha, depressão e isolamento social ${ }^{11}$. Ademais, sem tratamento, a bexiga neurogênica pode levar à sepse e insuficiência renal, o que impõe um alto custo ao sistema de saúde ${ }^{12}$.

Tendo em vista a relevância do cuidado no manejo da BN e IN, o presente estudo objetiva avaliar os efeitos de uma terapia comportamental associada a neuromodulação parassacral em indivíduos com doença de Parkinson.

\section{Metodologia}

\section{Desenho do estudo}

Trata-se de um estudo piloto do tipo ensaio clínico, em que sujeitos com DP nos estágios de 1 a 4 na escala de Hoehn \& Yahr foram submetidos à intervenção terapêutica na atenção às disfunções eliminatórias. 


\section{Local e período do estudo}

O estudo foi realizado de junho de 2019 a novembro de 2019, no serviço de Fisioterapia do Centro de Educação e Pesquisa em Saúde Anita Garibaldi (CEPS), na cidade de Macaíba, no Rio Grande do Norte.

\section{Amostra e elegibilidade}

A seleção dos participantes foi feita mediante os seguintes critérios de inclusão: indivíduos de ambos os gêneros com diagnóstico clínico de DP idiopática, de acordo com critérios do Banco de Cérebro de Londres (UK Parkinson's Disease Society Brain Bank clinical diagnostic criteria) $)^{13}$; estar classificado entre os estágios 1 e 4 da Escala de Incapacidade de Hoehn \& Yahr; apresentar queixas urinárias e diagnóstico de bexiga neurogênica pelo estudo urodinâmico; apresentar dois ou mais sintomas de constipação intestinal e estar em uso regular de medicações antiparkinsonianas. Foram utilizados como critérios de exclusão faltar 2 vezes consecutivas ao serviço; não completar as 16 sessões de tratamento e desistir, voluntariamente, de completar o protocolo e/ou retirar seu consentimento.

\section{Procedimentos de coleta de dados}

Todos os sujeitos realizaram uma entrevista semiestruturada, proposta pelos pesquisadores para coletar dados de identificação e sociodemográficos, uma avaliação clínica composta por questionários validados e estudo urodinâmico. Foram aplicados os questionários International Consultation Incontinence Questionnaire - Overactive Bladder (ICIQ-OAB), o questionário Parkinson's Disease Questionnaire (PDQ-39), Diário Miccional e Evacuatório e a Escala de Bristol Stool.

O ICIQ-OAB apresenta seis questões específicas sobre os sintomas miccionais - frequência diurna, noctúria, urgência e perda urinária durante a urgência, com suas correlações com a qualidade de vida. A versão em português do ICIQ-OAB foi traduzida, adaptada culturalmente e apresentou satisfatória confiabilidade e validade de constructo ${ }^{14}$. Para análise dos resultados, foram somados os valores correspondentes das questões $3 a, 4 a$, $5 a$ e $6 a$, obtendo um total de 0 a 16 pontos. Quanto maior o valor encontrado, maior o comprometimento.
Dessa forma, é recomendada a utilização desse questionário durante a investigação clínica ou científica dos sintomas miccionais relacionados à bexiga hiperativa. O Parkinson's Disease Questionnaire (PDQ-39), é composto por 39 itens e avalia o impacto da doença na qualidade de vida do indivíduo, com relação ao mês anterior, cuja versão brasileira já é validada ${ }^{15}$. 0 questionário é subdividido em oito dimensões: (1) Mobilidade (10 itens); (2) Atividade de vida diária (6 itens); (3) Bem estar emocional (6 itens); (4) Estigma (4 itens); (5) Suporte social (3 itens); (6) Cognição (4 itens); (7) Comunicação (3 itens) e (8) Desconforto corporal (3 itens).

Cada item pode ser respondido por meio de cinco opções e o escore varia entre 0 (nunca) e 4 (sempre ou impossível para mim). O escore por dimensão é calculado, de acordo, com a seguinte fórmula: $100 \mathrm{x}$ (soma dos escores / 4 x número de itens); e o escore total é a soma do resultado de cada dimensão dividido por 8 (total de dimensões). Os resultados, por dimensão e o total, apresentam-se em porcentagem.

A pontuação total varia entre 0 (nenhum problema) e 100 (máximo nível de problema), ou seja, uma baixa pontuação indica melhor percepção da qualidade de vida.

Os Diários Miccional e Evacuatório foram usados para avaliação das funções eliminatórias. Durante o preenchimento dos diários, os sujeitos foram orientados a anotar por 3 dias a frequência miccional (diurna e noturna), volume de líquido ingerido, volume urinado ou retirado do cateterismo intermitente, número de episódios de perda urinária, urgência miccional, bem como a quantidade de fraldas utilizadas diariamente ${ }^{16}$. Também foi orientado o preenchimento, por 7 dias, sobre as evacuações (frequência, necessidade de esforço e consistência das fezes, pela escala de Bristol). A Escala de Bristol para a consistência de fezes, traduzida e validada para o português para o uso no Brasil, apresentou alta confiabilidade, denotando sua utilidade na prática clínica ${ }^{17}$. A escala é descritiva e visual, e consta de sete tipos de fezes (fezes tipo 1 e 2 são ressecadas, 3 e 4 são normais e 5 a 5 são líquidas), sendo composta por imagens e suas respectivas definições; atendendo o propósito para o qual foi elaborada. 
Após o preenchimento dos questionários e posterior análise, identificando-se a presença de queixas, os sujeitos eram encaminhados para urologista do CEPS Anita Garibaldi e explicados sobre o Estudo Urodinâmico (EDU), o motivo pelo qual foi solicitado e todo o passo a passo para realização do exame.

Pelo estudo urodinâmico é possível identificar a causa dos sintomas urinários e quantificar os processos fisiopatológicos, também permite o diagnóstico da disfunção, orienta a escolha do tratamento apropriado e verificaa resposta ao tratamento, observando-se as fases de enchimento e esvaziamento da bexiga ${ }^{18}$.

No presente estudo, foram avaliadas as fases de enchimento e esvaziamento vesical, através da EDU com duração de 30 a 40 minutos. Foi dada ênfase principalmente aos valores da capacidade vesical e presença ou ausência de hiperatividade como desfechos. Após avaliados, todos os pacientes incluídos foram submetidos ao protocolo de intervenção com neuromodulação e terapia comportamental.

\section{Intervenção}

Foram realizados dois atendimentos por semana durante 8 semanas seguidas, totalizando 16 atendimentos, com duração de 40 minutos/cada. Na primeira sessão, o participante recebia uma explicação acerca da terapêutica comportamental e neuromodulação e era iniciado o protocolo. Segundo Benevento e Sipski em 2002 $\frac{19}{}$, a terapia comportamental compreende a análise da relação do sintoma do paciente e seu ambiente para modificação de maus hábitos miccionais e evacuatórios. Para esse estudo, a terapêutica envolveu educação do paciente em relação ao hábito urinário, reeducação vesical, estratégias para o controle do desejo miccional, posicionamento e treino evacuatório (o paciente deve ser estimulado a sentar-se no vaso sanitário, com os pés devidamente apoiados em suporte adequado à sua altura, durante cinco a dez minutos, após as principais refeições, tirando-se proveito do reflexo gastro-cólico), orientações para dieta e ingesta hídrica. As orientações foram realizadas de acordo com os resultados do diário miccional, evacuatório e estudo urodinâmico.

Posteriormente, foi explicada a Neuromodulação parassacral não invasiva e a colocação da mesma na terapia. Os participantes fizeram uso de um estimulador elétrico transcutâneo (Neurodyn Portátil/
Ibramed) com 2 eletrodos autoadesivos superficiais, de tamanho $5 \mathrm{~cm} \times 5 \mathrm{~cm}$, na região parassacral (S2-S4). A estimulação foi realizada com os parâmetros de largura de pulsos de $450 \mu$ s e frequência de $5 \mathrm{~Hz}$. A intensidade foi adaptada a cada indivíduo, mantendo-se a intensidade imediatamente abaixo do limiar que determina a contração. A neuromodulação foi realizada no CEPS e aplicada pelo terapeuta responsável, com duração de 30 minutos. Os 10 minutos restantes foram usados para reforçar a terapêutica comportamental e readequá-la quando necessário. Após a fase completa de intervenção, foi realizada reavaliação dos participantes com aplicação da ficha de avaliação, dos questionários previamente usados e estudo urodinâmico.

\section{Análise estatística}

Foram realizadas análises descritivas das variáveis estudadas a partir de frequência, medianas, médias e desvios-padrões. Para avaliar se a intervenção causaria aumento da capacidade vesical e melhora dos sintomas urinários, foi analisada a diferença das mesmas antes e depois pelo estudo urodinâmico, a partir de um teste não paramétrico de Wilcoxon. Como nível de significância estatística, considerou-se um $p \leq 0,05$, sendo os dados analisados pelo programa estatístico SPSS versão 20.0.

\section{Aspectos éticos}

O projeto de pesquisa foi aprovado pelo comitê de ética e pesquisa do Universidade Federal do Rio Grande do Norte-UFRN em junho de 2019, número de registro: CAAE:09905119.7.0000.5537. Os pacientes assinaram o TCLE para participar da pesquisa.

\section{Resultados}

Foram avaliados 19 pacientes com DP idiopática e com queixas urinárias e intestinais, entretanto, desses, 15 confirmaram diagnóstico de bexiga neurogênica com a urodinâmica e no decorrer do estudo, ocorreram 9 perdas/exclusões (Figura 1). A amostra final compôsse de 6 pacientes de ambos os sexos, sendo a maioria homens $(66,67 \%)$ apresentando variações de estágio e tempo da doença (Tabela 1). Após as 16 sessões, foi observada melhora dos sintomas urinários, segundo o ICIQ-OAB, para todos os pacientes, apresentando variação da média de 9,33 $( \pm 3,5)$ para 4,66 $( \pm 3,07)$ com incremento significativo dos valores $(P=.027)$. 
Quanto aos escores de qualidade de vida específico para doença de Parkinson (PDQ-39), houve uma redução do escore total em $66,67 \%$ da amostra. Em relação aos domínios, houve melhora nos valores em 6 dos 8 domínios avaliados conforme apresentado na Tabela 2.

No estudo urodinâmico, por meio da cistometria, houve melhora nos valores da capacidade vesical que, embora não significantes, foram evidenciados em cerca de $83,3 \%$ dos pacientes. No que se refere à hiperatividade pode-se observar melhora dos níveis de hiperatividade em 5 dos 6 pacientes em que dois deles não apresentavam mais nenhum momento de hiperatividade durante a cistometria por meio do estudo urodinâmico. (Tabela 3).

Já em relação aos aspectos de eliminação evacuatória medido pelo diário evacuatório, 5 dos participantes apresentaram melhora do quadro no que se refere à frequência, uma vez que uma das participantes se manteve com a evacuação diária que já possuía em sua rotina. No quesito esforço ao evacuar, 100\% dos participantes referiram melhora, não mais necessitando de esforço para iniciar a evacuação.

$\mathrm{Na}$ avaliação dos aspectos das fezes aferidos com a Escala de Bristol, antes da intervenção 33,33\% da amostra, apresentavam fezes tipo 1, 16,66\% fezes tipo 2,33,33\% apresentavam fezes tipo 3 e 16,66\% fezes tipo 5. Após a intervenção, 16,66\% apresentaram fezes tipo 2, 66,66\% fezes tipo 3 e 16,66\% apresentaram fezes tipo 4. (Tabela 4).

Figura 1. Fluxograma de consituição da amostra
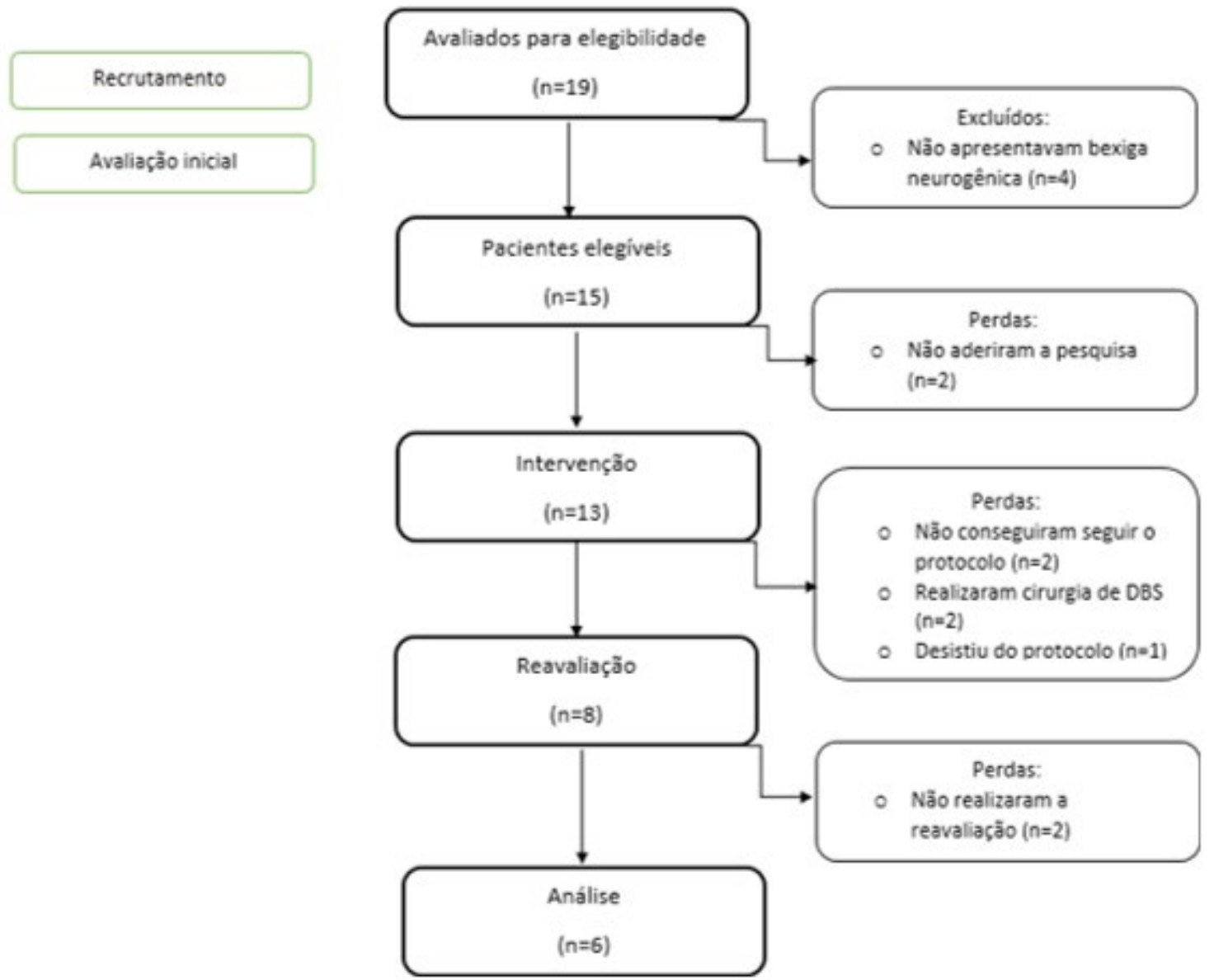
Tabela 1. Caracterização da amostra

\begin{tabular}{cc}
\hline Sexo $(\mathrm{N}=6)$ & $\mathrm{N}(\%)$ \\
\hline Feminino & $2(33,3 \%)$ \\
Masculino & $4(66,6 \%)$ \\
\hline Hoehn \&Yahr & \\
\hline 1 & $2(33,6 \%)$ \\
2 & $1(16,6 \%)$ \\
3 & $2(33,6 \%)$ \\
4 & $1(16,6 \%)$ \\
\hline Tempo de Doensa & \\
(anos) & $2(33,6)$ \\
\hline 4 & $1(16,6 \%)$ \\
5 & $2(33,6 \%)$ \\
10 & $1(16,6 \%)$
\end{tabular}

Tabela 2. Escores e variações do PDQ-39, por domínio, para os participantes

\begin{tabular}{|c|c|c|c|c|c|c|c|c|c|c|c|c|c|c|c|c|}
\hline \multirow{2}{*}{$\begin{array}{l}\text { Dominios } \\
\text { Paciente } \\
\end{array}$} & \multicolumn{2}{|c|}{ Mobilidade } & \multicolumn{2}{|c|}{ AVD } & \multicolumn{2}{|c|}{ Bem estar emocional } & \multicolumn{2}{|c|}{ Estigma } & \multicolumn{2}{|c|}{ Suporte social } & \multicolumn{2}{|c|}{ Cognição } & \multicolumn{2}{|c|}{ Comunicação } & \multicolumn{2}{|c|}{ Desconforto corporal } \\
\hline & Antes & Depois & Antes & Depois & Antes & Depois & Antes & Depois & Antes & Depois & Antes & Depois & Antes & Depois & Antes & Depois \\
\hline 1 & 7,75 & 8,25 & 70,83 & 75 & 70,83 & 62,5 & 70,83 & 31,25 & 70,83 & 41,66 & 70,83 & 31,25 & 70,83 & so & 70,83 & 50 \\
\hline 2 & 1,5 & 1,25 & 25 & 25 & 41,66 & 45,83 & 6,25 & 6,25 & 16,66 & 25 & 18,75 & 31,25 & 33,33 & 33,33 & 66,66 & 58,33 \\
\hline 3 & 2,75 & 1,5 & 37,5 & 58,33 & 16,66 & 25 & 0 & 25 & 0 & 0 & 25 & 12,5 & 16,66 & 16,66 & 50 & 50 \\
\hline 4 & 42,5 & 15 & 50 & 37,5 & 79,16 & 83,33 & 18,75 & 6,25 & 25 & 33,33 & 75 & 37,5 & 0 & 8,33 & 41,66 & 50 \\
\hline 5 & 7,75 & 7 & 70,83 & 70,83 & 54,16 & 54,16 & 62,5 & 62,5 & 16,66 & 16,66 & so & so & 83,33 & 83,33 & 66,66 & 66,66 \\
\hline 6 & 0 & 0 & 0 & 0 & 4,16 & 0 & 6,25 & 6,25 & 0 & 0 & 12,5 & 0 & 0 & 0 & 0 & 0 \\
\hline $\begin{array}{c}\text { Total } \\
\text { (média) }\end{array}$ & 10,37 & 5.5 & 42,36 & 44,44 & 44,43 & 45,13 & 27,43 & 22,91 & 21.52 & 19.44 & 42,01 & 27,08 & 34,02 & 31,94 & 49,3 & 45,83 \\
\hline
\end{tabular}

Tabela 3. Resultado para capacidade cistométrica máxima antes e após a intervenção

\begin{tabular}{|c|c|c|}
\hline \multirow[b]{2}{*}{ Paciente } & \multicolumn{2}{|c|}{ Máxima } \\
\hline & Antes & Depois \\
\hline 1 & 448 & 484 \\
\hline 2 & 196 & 296 \\
\hline 3 & 266 & 162 \\
\hline 4 & 95 & 470 \\
\hline 5 & 132 & 142 \\
\hline 6 & 115 & 190 \\
\hline
\end{tabular}

\section{Discussão}

Bexiga e intestino neurogênicos são condições comuns na doença de Parkinson. Com relação ao tratamento, embora várias intervenções estejam disponíveis, há poucas evidências de que estas melhorem os resultados para essa população. Porém, sugere-se que sejam realizadas, primariamente, abordagens conservadoras ${ }^{6}$.

Dentre essas abordagens estão as intervenções farmacológicas, especialmente medicamentos anticolinérgicos, mostrando-se como opção de primeira linha no tratamento ${ }^{6}$. No presente estudo, a intervenção medicamentosa não foi a primeira escolha terapêutica devido o histórico de polifarmácia apresentado pelos pacientes, possíveis efeitos colaterais em especial o agravo da constipação e consequente interação medicamentosa o que poderia ser motivo de viés de aferição da terapêutica de escolha. Ademais, um estudo de Palleschi G et al. avaliou e tratou a bexiga neurogênica com medicamentos antimuscarínicos de 39 pacientes, dos quais 7 apresentavam DP e 20\% destes abandonaram o protocolo devido a constipação, tontura/dor de cabeça e nenhuma melhora clínica ${ }^{20}$. 
Nesse estudo, avaliou-se os efeitos do tratamento da terapia comportamental associada à neuromodulação, que também são opções conservadoras para o manejo da BN e IN. Dois estudos pilotos já foram conduzidos no campo da terapia comportamental, entretanto, eles consideraram como terapêutica comportamental exercícios para músculos do assoalho pélvico usando biofeedback e apresentaram alto risco de viés e baixa clareza ${ }^{6}$. Para esse estudo, foi considerado como terapêutica comportamental as orientações quanto posicionamento ao evacuar, técnica de respiração diafragmática, ingestão hídrica adequada, massagem terapêutica abdominal e prática regular de exercícios físicos, uma vez que todos os participantes estavam inseridos em fisioterapia convencional.

A terapia comportamental foi associada ao uso da neuromodulação por duas vezes na semana, durante 30 minutos, o uso dessa técnica vem crescendo como uma opção terapêutica. Em um estudo prospectivo com 10 pacientes com DP, foi estimulado o nervo tibial anterior, e observou-se pequenas melhorias, apesar de estatisticamente insignificantes, para frequência de micção, episódios de incontinência e urgência ${ }^{6}$.

No presente estudo foi usado a eletroestimulação parassacral, isso porque a neuromodulação parassacral tende a estimular diretamente os reflexos espinhais e alcançar centros supraespinhais em uma intensidade mais eficaz do que o nervo tibial posterior, que é mais periférico ${ }^{21}$. A neuromodulação do nervo sacral, realizada por meio de implante de eletrodos no forame sacral (s3), mostrou-se capaz de modular estruturas corticais e subcorticais importantes envolvidas na consciência e alerta, sensação de enchimento da bexiga e o momento da micção, levando a uma inibição do reflexo da micção em pacientes adultos com incontinência urinária. Esse efeito benéfico origina-se das áreas supraespinhais do cérebro, que são inibidas ou ativadas pela porção caudal da medula espinal durante a estimulação, como evidenciado no estudo de Blok et al. (apud Sacomani e Goldman) ${ }^{10}$.

Nesse estudo evidenciou que a associação dessas terapias foi estatisticamente significativa quando investigada a gravidade dos sintomas miccionais relacionados à bexiga hiperativa pelo ICIQ-OAB. Kabay et al, o estudarem o efeito da estimulação percutânea do nervo tibial posterior em 47 pacientes com DP numa intervenção de 12 semanas, também observaram melhoras significativas 22 .

Quanto ao escores de qualidade de qualidade de vida específicos para DP, houve melhora em 6 dos 8 domínios avaliados. Destaca-se a melhora no domínio mobilidade, uma vez que Bolaños, em 2019, investigou a importância dos sintomas não motores para quedas em pacientes com DP. Uma possível explicação para a associação entre sintomas urinários e quedas é a noctúria, que geralmente é o fator predominante que leva à queda noturna. Esses achados sugerem a necessidade de uma abordagem mais intensiva na identificação e tratamento dos sintomas urinários em pessoas com DP 23 .

Quanto ao estudo urodinâmico, Vurture et al. ${ }^{24}$ observaram os achados urodinâmicos em pacientes com (DP) com sintomas de bexiga hiperativa. Foram incluídos 42 pacientes com duração média da DP no momento do EU de 6,6 anos. Em todos foi encontrada hiperatividade do detrusor e com média de capacidade cistométrica máxima de $274,8 \mathrm{~mL}( \pm 174,3)$. Esses resultados corroboram com os achados do presente estudo a avaliação inicial, em que todos os participantes apresentaram hiperatividade detrusora e baixa capacidade cistométrica máxima.

Um estudo de Ohannessian et al. que avaliou os efeitos da neuromodulação com estimulação do nervo tibial anterior, propôs um tratamento que consistiu em uma sessão de 20 minutos por dia durante seis semanas. Dos seis pacientes com DP avaliados com quadro de bexiga hiperativa, pode-se observar que não houve diferença significativa nos parâmetros urodinâmicos, apesar de apresentarem uma tendência de melhora para capacidade cistométrica máxima ${ }^{25}$, reforçando os dados encontrados no presente estudo.

Já em relação aos aspectos evacuatórios, os achados iniciais foram uma baixa frequência evacuatória, com necessidade de esforço e consistência dura para fezes. Sabe-se que a constipação não é um problema apenas do intestino, pois pode também levar a problemas de controle da bexiga. Quando o reto está cheio de fezes, por exemplo, pode ocasionar um distúrbio na bexiga e provocar a sensação de urgência e frequência ${ }^{26}$, desse modo é importante avaliar e tratar esses pacientes. 
Numa revisão sistemática, Knudsen et al, observou, ao reunir os resultados dos estudos mais homogêneos na DP, uma prevalência de constipação em $40 \%$ a 50\%, considerando-se constipação uma frequência de menos de 3 evacuações por semana ou presença de esforço; apesar da grande variação entre os estudos individuais. Além disso, observou-se um claro aumento na prevalência de constipação relacionada ao aumento do estágio clínico da doença²7.

Um estudo de Mukherjee, Biswas e Das afirmou que o tratamento começa com dieta rica em fibras, ingestão adequada de líquidos e fisioterapia. Entretanto, o manejo dessa condição pode ser complicado, pois inclui não apenas o tratamento sintomático, mas também a otimização de medicamentos antiparkinsonianos, principalmente anticolinérgicos e dopaminérgicos. Estudos sobre novos agentes terapêuticos e intervenções não farmacoterapêuticas seriam úteis ${ }^{28}$.

Com a intervenção proposta no estudo, observou-se melhora na frequência evacuatória e consistência das fezes, sem necessidade de esforço; mostrando-se, portanto, uma alternativa positiva para o tratamento.

Em se tratando de um estudo piloto, o presente estudo possui limitações inerentes ao seu desenho não randomizado, bem como o tamanho da amostra relativamente pequeno e a falta de poder estatístico, fatores estes que impediram a avaliação com maior poder inferencial dos fatores preditivos de sucesso para a intervenção.

Apesar dos resultados encorajadores desse estudo, mais pesquisas são necessárias para avaliar os efeitos da neuromodulação na atenuação destes sintomas na DP. Estudos adicionais mais amplos, bem projetados e randomizados com foco no tratamento requerem investigações relevantes e manejo adequado das disfunções miccionais e evacuatórias para meIhorar a qualidade de vida e proteger o trato urinário superior das pessoas com doença de Parkinson.

\section{Conclusão}

Pelo presente estudo, pode-se concluir que a terapêutica comportamental acrescida da neuromodulação parassacral atua na melhora dos sintomas urinários e da condição evacuatória. O presente estudo sugere continuidade do mesmo, uma vez que pode- se evidenciar que o protocolo instituído acarretou mudanças positivas nos valores da qualidade de vida (nos domínios de mobilidade, estigma, suporte social, cognição, comunicação e desconforto corporal) bem como na capacidade cistométrica e na hiperatividade.

\section{Contribuições dos autores}

Azevedo MAR participou da concepção, delineamento, busca e análise estatística dos dados da pesquisa, interpretação dos resultados e redação do artigo científico do artigo. Medeiros CEB participou da coleta e interpretação dos dados. Gonçalves RP participou da avaliação e coleta de dados. Almeida VA participou da coleta de dados. Lisboa LL participou do delineamento, análise estatística, interpretação dos resultados e redação.

\section{Conflitos de interesses}

Nenhum conflito financeiro, legal ou político envolvendo terceiros (governo, empresas e fundações privadas, etc.) foi declarado para nenhum aspecto do trabalho submetido (incluindo, mas não se limitando a subvenções e financiamentos, participação em conselho consultivo, desenho de estudo, preparação de manuscrito, análise estatística, etc.).

\section{Referências} 1. Jankovic J. Parkinson's disease: clinical features and diagnosis.
J. Neurol. Neurosurg. Psychiatry. 2008;79(4):368-76. http://dx.doi.
org/10.1136/jnnp.2007.131045

2. Pfeiffer RF. Non-motor symptoms in Parkinson's disease. Parkinsonism Relat Disord. 2016;22(supl 1):119-22. http://dx.doi. org/10.1016/j.parkreldis.2015.09.004

3. Jellinger KA. Neuropathobiology of non-motor symptoms in Parkinson disease. J Neural Transm. 2015;122(10):1429-40. https://doi.org/10.1007/s00702-015-1405-5

4. Chen H, Zhao EJ, Zhang W, Lu Y, Liu R, Huang X, et al. Metaanalyses on prevalence of selected Parkinson's nonmotor symptoms before and after diagnosis. Transl Neurodegeneration. 2015;4(1):1-10. http://dx.doi.org/10.1186/2047-9158-4-1

5. Winge K, Fowler CJ. Bladder dysfunction in Parkinsonism: Mechanisms, prevalence, symptoms, and management. J Mov. Disord. 2006;21(6):737-45. http://dx.doi.org/10.1002/mds.20867

6. Hajebrahimi S, Chapple CR, Pashazadeh F, Salehi-Pourmehr H. Management of neurogenic bladder in patients with Parkinson's disease: A systematic review. Neurourol Urodyn. 2018;38(1):31-62. http://dx.doi.org/10.1002/nau.23869 
7. Sakakibara R, Shinotoh H, Uchiyama T, Sakuma M, Kashiwado $M$, Yoshiyma, et al. Questionnaire-based assessment of pelvic organ dysfunction in Parkinson's disease. Auton Neurosci. 2001;92(1-2):76-85. http://dx.doi.org/10.1016/s15660702(01)00295-8

8. Pablo-Fernández E, Passananti V, Zárate-López N, Emmanuel A, Warner T. Colonic transit, high-resolution anorectal manometry and MRI defecography study of constipation in Parkinson's disease. Parkinsonism Relat Disord. 2019;66:195-201. http:// dx.doi.org/10.1016/j.parkreldis.2019.08.016

9. Batla A, Tayim N, Pakzad M, Panicker JN. Treatment Options for Urogenital Dysfunction in Parkinson's Disease. Cur Treat Options Neurol. 2016;18(10):45. https://doi.org/10.1007/s11940-016-0427$\underline{0}$

10. Sacomani CAR, Goldman H. Visão Geral das Estratégias de Tratamento: Eletroneuroestimulação e eletroneuromodulação. In: Rios LAS, Averbeck MA, Madersbacher H, editores. Neurourologia: Manual para prática clínica. São Paulo: Sociedade Brasileira de Urologia; 2017. p. 102-8.

11. O'Leary M, Dierich M. Botulinum toxin type A for the treatment of urinary tract dysfunction in neurological disorders. Urol Nurs. 2010;30(4):228-34. Citado em: PMID: 20949807

12. Dorsher PT, McIntosh PM. Neurogenic bladder. Adv Urol. 2012:816274. https://doi.org/10.1155/2012/816274

13. Hughes A J, Daniel SE, Kilford L, Lees AJ. Accuracy of clinical diagnosis of idiopathic Parkinson's disease: a clinico-pathological study of 100 cases. Journal Of Neurology, Neurosurgery \& Psychiatry. 1992;55(3):181-184. http://dx.doi.org/10.1136/ jnnp.55.3.181

14. Pereira SB, Thiel RRC, Riccetto C, Silva JM, Pereira LC, Herrmann V, et al. Validação do International Consultation on Incontinence Questionnaire Overactive Bladder (ICIQ-OAB) para a língua portuguesa. Rev. Bras. Ginecol. Obstet. 2010;32(6):273-8. http://dx.doi.org/10.1590/s0100-72032010000600004

15. Carod-Artal FJ, Martinez-Martin P, Vargas AP. Independent validation of SCOPA-psychosocial and metric properties of the PDQ-39 Brazilian version. J Mov. Disord. 2007;22(1):91-8. https:// doi.org/10.1002/mds.21216

16. Feldner Junior PC. Diagnóstico clínico e subsidiário da incontinência urinária. Rev. Bras. Ginecol. Obstet. 2006;28(1):5462. https://doi.org/10.1590/S0100-72032006000100010

17. Martinez AP, Azevedo GR. Tradução, adaptação cultural e validação da Bristol Stool Form Scale para a população brasileira. Rev. Latino-am. Enfermagem. 2012;4(20):1-7. https://doi. org/10.1590/S0104-11692012000300021
18. Costa Monteiro LM. Mielomeningocele. In: D'Ancona CAL, Netto NR Jr, organizadores. Aplicações Clínicas da Urodinâmica.

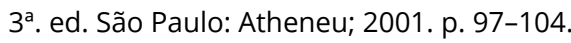

19. Benevento BT, Sipski ML. Neurogenic bladder, neurogenic bowel, and sexual dysfunction in people with spinal cord injury. Physical therapy. 2002;82(6):601-612. Ciatdo em: PMID: 12036401

20. Palleschi G, Pastore AL, Stocchi F, Bova G, Inghilleri M, Sigala S, et al. Correlation Between the Overactive Bladder Questionnaire (OAB-q) and Urodynamic Data of Parkinson Disease Patients Affected by Neurogenic Detrusor Overactivity During Antimuscarinic Treatment. Clin Neuropharmacol. 2006;29(4):2209. http://dx.doi.org/10.1097/01.wnf.0000228177.75711.0f

21. Barroso U, Viterbo W, Bittencourt J, Farias T, Lordêlo $P$. Posterior Tibial Nerve Stimulation vs Parasacral Transcutaneous Neuromodulation for Overactive Bladder in Children. J. Urol. 2013;190(2):673-7. http://dx.doi.org/10.1016/j.juro.2013.02.034

22. Kabay S, Kabay SC, Cetiner M, Mestan E, Sevim M, Ayas S, et al. The Clinical and Urodynamic Results of Percutaneous Posterior Tibial Nerve Stimulation on Neurogenic Detrusor Overactivity in Patients With Parkinson's Disease. Urology. 2016;87:76-81. http:// dx.doi.org/10.1016/j.urology.2015.09.026

23. Alvarado-Bolaños A, Cervantes-Arriaga A, Arredondo-Blanco K, Salinas-Barboza K, Isais-Millán S, Rodríguez-Violante M. Falls in persons with Parkinson's disease: Do non-motor symptoms matter as much as motor symptoms?. Arq Neuro-psiquiatr. 2017;77(11):761-7. http://dx.doi.org/10.1590/0004-282x20190148

24. Vurture G, Peyronnet B, Palma JÁ, Sussman RD, Malacarne DR, Felgin Andrew, et al. Urodynamic Mechanisms Underlying Overactive Bladder Symptoms in Patients With Parkinson Disease. Int Neurourol J. 2019;23(3):211-8. http://dx.doi.org/10.5213/ inj.1938086.043

25. Ohannessian A, Kaboré FA, Agostini A, Aurier KL, Witjas T, Azulay JP, et al. Stimulation transcutanée chronique du nerf tibial dans l'hyperactivité vésicale des syndromes parkinsoniens. Prog. Urol. 2013;23(11):936-9. http://dx.doi.org/10.1016/j. purol.2013.07.004

26. Palma PCR, editor. Urofisioterapia: Aplicações Clínicas das Técnicas Fisioterapêuticas nas Disfunções Miccionais e do Assoalho Pélvico. Campinas: Personal Link Comunicações Ltda.; 2009.

27. Knudsen K, Krogh K, Ostergaard K, Borghammer. Constipation in parkinson's disease: Subjective symptoms, objective markers, and new perspectives. Mov Disord. 2017;32(1):94-105. https://doi. org/10.1002/mds.26866

28. Mukherjee A, Biswas A, Das S. Gut dysfunction in Parkinson's disease. World J Gastroenterol. 2016;22(25):5742-74. http://dx.doi. org/10.3748/wjg.v22.i25.5742 